\title{
Novel Complex Unbalanced Dicentric $X$-Autosome Rearrangement in a Thoroughbred Mare with a Mild Effect on the Phenotype
}

\author{
Mayra N. Mendoza ${ }^{a}$ Sam A. Schalnus ${ }^{b}$ Bitsy Thomson ${ }^{b}$ Rebecca R. Bellone \\ Rytis Juras ${ }^{d}$ Terje Raudsepp ${ }^{d}$ \\ aEstación Experimental Agraria Chincha, Dirección de Recursos Genéticos y Biotecnología, Instituto Nacional de

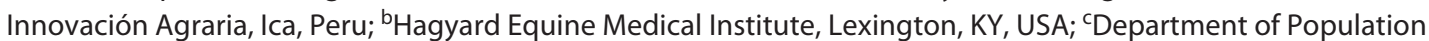 \\ Health and Reproduction, Veterinary Genetics Laboratory, School of Veterinary Medicine, University of California, \\ Davis, CA, USA; ${ }^{\mathrm{d}}$ Molecular Cytogenetics Laboratory, College of Veterinary Medicine and Biomedical Sciences,Texas \\ A\&M University, College Station, TX, USA
}

\section{Keywords \\ Complex rearrangement - Dicentric $\cdot$ Horse $\cdot$ Unbalanced . $\mathrm{X}$-autosome translocation}

\begin{abstract}
Complex structural $\mathrm{X}$ chromosome abnormalities are rare in humans and animals, and not recurrent. Yet, each case provides a fascinating opportunity to evaluate $\mathrm{X}$ chromosome content and functional status in relation to the effect on the phenotype. Here, we report the first equine case of a complex unbalanced X-autosome rearrangement in a healthy but short in stature Thoroughbred mare. Studies of about 200 cells by chromosome banding and FISH revealed an abnormal $2 n=63, \mathrm{X}$, der $(\mathrm{X} ; 16)$ karyotype with a large dicentric derivative chromosome (der). The der was comprised of normal Xp material, a palindromic duplication of Xq12q21, and a translocation of chromosome 16 to the inverted Xq12q21 segment by the centromere, whereas the distal Xq22q29 was deleted from the der. Microsatellite genotyping determined a paternal origin of the der. While there was no option to experimentally investigate the status of $\mathrm{X}$ chromosome inactivation $(\mathrm{XCl})$, the observed mild phenotype of this case suggested the following scenario to retain an almost normal
\end{abstract}

genetic balance: active normal $\mathrm{X}$, inactivated $\mathrm{X}$-portion of the der, but without $\mathrm{XCl}$ spreading into the translocated chromosome 16. Cases like this present unique resources to acquire information about species-specific features of $X$ regulation and the role of X-linked genes in development, health, and disease.

(c) 2020 S. Karger AG, Basel

\section{Introduction}

Numerical and structural abnormalities of the X chromosome in humans and animals are often compatible with life, particularly in females [Ciaccio et al., 2020; Raudsepp and Chowdhary, 2016]. This is largely attributed to random $\mathrm{X}$ chromosome inactivation (XCI) in mammalian females [Lyon, 1961, 1962], which balances $\mathrm{X}$ chromosome gene dosage between sexes but also buffers deleterious effects of X chromosome mutations [Cantone and Fisher, 2017; Carrel and rown, 2017]. The process of XCI is triggered by cis-spreading of a long, noncoding RNA (lncRNA) transcribed from the X-linked XIST gene [Disteche, 1999; Deng et al., 2014]. Due to the cis-action of XIST, in cases of X-autosome rearrange- karger@karger.com

www.karger.com $/ \mathrm{cgr}$

(C) 2020 S. Karger AG, Base

Karger'
Molecular Cytogenetics Laboratory, Veterinary Integrative Biosciences College of Veterinary Medicine and Biomedical Sciences, Texas A\&M University 588 Raymond Stotzer Pkwy, College Station TX 77843-4458 (USA)

traudsepp@cvm.tamu.edu 
ments, inactivation can also spread to autosomes [Loda et al., 2017]. However, under normal conditions, not all genes undergo XCI [Abrams and Cotter, 2004; Alexandrou et al, 2016]. These include the pseudoautosomal genes, gametologs, and an additional set of dosage sensitive X-linked genes [Bellott et al., 2014; alaton and rown, 2016; Tukiainen et al., 2017]. Haploinsufficiency or overdose for these genes due to $\mathrm{X}$ chromosome rearrangements, particularly aneuploidies, may affect normal development and viability [Bellott et al., 2014]. Functional compartmentalization of the $\mathrm{X}$ chromosome into regions that undergo $\mathrm{XCI}$ and those that escape also means that genetic consequences of $\mathrm{X}$ chromosome rearrangements depend on which regions are involved. Furthermore, the extent of XCI varies between species [Berletch et al., 2015; Tukiainen et al., 2017], implying that homologous X chromosome aberrations may have different phenotypic effects in different species [Raudsepp et al., 2012; Raudsepp and Chowdhary, 2016].

In humans, the most common $\mathrm{X}$ chromosome rearrangements are aneuploidies with the reported frequency of X trisomy $(47, \mathrm{XXX})$ and X monosomy $(45, \mathrm{X}$; also known as Turner syndrome) of 1:1,000 and 1:2,500 live female births, respectively [Skuse et al., 2018]. Likewise, over $90 \%$ of all reported chromosome abnormalities in the horse (Equus caballus, ECA; $2 n=64$ ) involve the X chromosome, of which X monosomy $(63, \mathrm{X})$ makes up almost a half [Bugno et al., 2007; Lear and Bailey, 2008; Lear and Villagomez, 2011; Raudsepp et al., 2012; Raudsepp and Chowdhary, 2016]. Compared to aneuploidies, reports about structural aberrations involving the $\mathrm{X}$ chromosome are less common. Of these, rearrangements resulting in the loss of the entire short arm either by Xp deletion or due to the formation of isochromosomes of the long arm $\mathrm{i}(\mathrm{Xq})$ typically have phenotypes similar to $\mathrm{X}$ monosomy as reported in humans [Haltrich et al., 2015; Skuse et al., 2018] and horses [Lear and Bailey, 2008; Lear and Villagomez, 2011]. Other types of X chromosome structural rearrangements, however, are rare in both species. The frequency of balanced and unbalanced X-autosome translocations in humans is about 1:30,000 live births [Watanabe et al., 2018] with about a hundred cases described to date [Schmidt and Du Sart, 1992; Prueitt et al., 2000; Abrams and Cotter, 2004; Moyses-Oliveira et al., 2015; Watanabe et al., 2018]. In animals, only a handful of cases of X-autosome translocations have been reported in pigs [Koykul et al., 2000; Szczerbal and Switonski, 2016] and cattle [Gustavsson et al., 1968; Basrur et al., 2001], and just 2 cases in the horse [Power, 1987; BugnoPoniewierska et al., 2018]. However, more complex X chromosome rearrangements with multiple breakpoints are extremely rare, with just a few cases so far reported in humans [Haltrich et al., 2015].

Here, we describe a novel complex unbalanced X chromosome rearrangement combined with $\mathrm{X}$-autosome translocation in a Thoroughbred mare. The highly rearranged derivative chromosome (der) is delineated by GTG- and CBG-banding, FISH analysis, and short tandem repeat (STR) genotyping. Possible genetic consequences of the rearrangement are discussed. The presented rearrangement is the first of its kind found in the horse and adds to our understanding of X chromosome regulation in this species.

\section{Material and Methods}

\section{Case Description}

A 2-year-old Thoroughbred mare was referred to the Texas A\&M University Molecular Cytogenetics Laboratory for chromosome analysis because of short stature. At this age, the horse stood from ground up to the highest point of the withers 13.5 hands (137 $\mathrm{cm}$ ), which is abnormally short for the breed [Green, 1976]. Otherwise, the animal had been healthy through her life and was successfully used for riding and as a companion. However, there was no information about fertility, because her reproductive tract was never examined and, to the best of our knowledge, no attempts have been made to breed her.

\section{Cell Culture and Chromosome Preparation}

Short-term blood lymphocyte cultures and chromosome preparations followed standard procedures described elsewhere [Raudsepp and Chowdhary, 2008]. Briefly, $1 \mathrm{~mL}$ of sodium heparin stabilized peripheral blood was grown for $72 \mathrm{~h}$ in $9 \mathrm{~mL}$ of culture medium containing RPMI-1640 with HEPES and Glutamax (Gibco), $10 \%$ fetal bovine serum, $1 \times$ antibiotic-antimycotic (100×; Invitrogen, USA), and $15 \mu \mathrm{g} / \mathrm{mL}$ pokeweed mitogen (Sigma Aldrich). The cells were harvested with $10 \mu \mathrm{g} / \mathrm{mL}$ demecolcine solution (Sigma Aldrich), treated with optimal hypotonic solution (Rainbow Scientific), and fixed in 3:1 methanol/acetic acid. The cells were dropped on pre-cleaned wet glass slides and air dried. The quality of chromosome preparations was examined under a light microscope with $20 \times$ phase contrast objective.

\section{Karyotyping and Cytogenetic Analysis}

Chromosomes were stained with Giemsa for initial analysis of chromosome number and morphology. For refined analysis and karyotyping, the chromosomes were stained by GTG-banding [Seabright, 1971], and the X chromosome was further studied by CBG-banding [Arrighi and Hsu, 1971]. A minimum of 20 cells were captured and analyzed for each technique using an Axioplan2 microscope (Carl Zeiss, Inc., Jena, Germany) and IKAROS (MetaSystems GmbH, Altlussheim, Germany) software. The chromosomes were identified and arranged in a karyotype according to the International System of Cytogenetic Nomenclature of the Domestic Horse [Bowling et al., 1997]. 
Table 1. FISH markers used to dissect the complex rearrangement

\begin{tabular}{|c|c|c|c|c|c|}
\hline FISH probe/BAC & BAC sequence, EquCab3 & $\begin{array}{l}\text { Cytogenetic } \\
\text { location }\end{array}$ & Reference marker & Status in der & FISH mapping reference \\
\hline 148G5 & $16: 6,263,662-6,447,094$ & $16 \mathrm{q} 12 \mathrm{q} 13$ & PPARG & Present & Raudsepp et al. [2008] \\
\hline $206 \mathrm{~L} 9$ & $16: 80,991,274-81,133,927$ & $16 \mathrm{q} 24$ & PLOD2 & Present & Raudsepp et al. [2008] \\
\hline $194 \mathrm{E} 12$ & $X: 3,945-246,703$ & $\mathrm{Xp} 24 \mathrm{p} 25$ & PLCXD1 & Present & $\begin{array}{l}\text { Raudsepp and Chowdhary } \\
\text { [2008] }\end{array}$ \\
\hline 288L23 & $\mathrm{X}: 24,982-287,625$ & $\mathrm{Xp} 24 \mathrm{p} 25$ & SHOX & Present & $\begin{array}{l}\text { Raudsepp and Chowdhary } \\
\text { [2008] }\end{array}$ \\
\hline $121 \mathrm{H} 14$ & $X: 46,234,722-46,443,852$ & $\mathrm{Xp} 12 \mathrm{p} 11$ & KLF8 & Present & This study \\
\hline ECAcons70; PCR product & na & Centromeres & na & Present & Alkan et al. [2011] \\
\hline 279 A6 & $X: 49,788,996-49,959,217$ & $\mathrm{Xq11q} 12$ & $A M E R 1$ & Duplicated/inverted & This study \\
\hline 06D24 & $X: 52,786,432-52,963,819$ & $\mathrm{Xq12q13}$ & $A R$ & Duplicated/inverted & Raudsepp et al. [2008] \\
\hline $155 \mathrm{G} 10$ & $\mathrm{X}: 58,215,815-58,432,305$ & $\mathrm{Xq13}$ & $\begin{array}{l}\text { LOC102150010 } \\
\text { (likely XIST) }\end{array}$ & Duplicated/inverted & Raudsepp et al. [2004] \\
\hline $194 \mathrm{~K} 23$ & $X: 68,878,122-69,076,409$ & $\mathrm{Xq} 16$ & $\mathrm{CHM}$ & Duplicated/inverted & Raudsepp et al. [2004] \\
\hline ETSTY7 PCR product & na & $\mathrm{Xq} 17$ and $\mathrm{Yq}$ & $\begin{array}{l}\text { ETSTY7; } \\
\text { ampliconic array }\end{array}$ & Duplicated/inverted & Janecka et al. [2018] \\
\hline $125 \mathrm{~B} 23$ & $\mathrm{X}: 83,050,951-83,248,364$ & $\mathrm{Xq} 21$ & PCDH19 & Duplicated/inverted & This study \\
\hline 030D8 & $\mathrm{X}: 87,857,818-88,044,827$ & $\mathrm{Xq} 22$ & SERPINA7 & Deleted & This study \\
\hline $072 J 3$ & $\mathrm{X}: 89,900,666-90,131,458$ & $\mathrm{Xq} 23$ & IRS4 & Deleted & Raudsepp et al. [2004] \\
\hline $332 \mathrm{H} 13$ & $X: 95,555,675-95,752,571$ & $\mathrm{Xq} 24$ & PLS3 & Deleted & This study \\
\hline 089G8 & X:99,194,129-99,440,647 & $\mathrm{Xq} 26$ & $L A M P 2$ & Deleted & Raudsepp et al. [2004] \\
\hline 194D2 & $X: 126,548,219-126,768,411$ & $\mathrm{Xq} 28$ & TEX28 & Deleted & This study \\
\hline
\end{tabular}

Selection of Markers for Molecular Cytogenetic Analysis

We used information of the equine cytogenetic map [Raudsepp et al., 2004, 2008], the horse X chromosome sequence map in the recent genome assembly EquCab3 [Kalbfleisch et al., 2018], and the corresponding CHORI-241 BAC clone track in NCBI Genome (https://www.ncbi.nlm.nih.gov/genome/) to select markers for molecular cytogenetic analysis. These included $2 \mathrm{BAC}$ clones from ECA16 and 13 BACs from the X chromosome (Table 1). The latter were selected along ECAX to cover the most distal and most proximal parts of the short $(\mathrm{Xp})$ and the long $(\mathrm{Xq})$ arms, regions involved in the duplication/inversion and regions around the putative breakpoint. In addition, we used 2 PCR products as probes, one obtained with primers for horse centromeric consensus sequences ECAcons70 [Alkan et al., 2011] and the other corresponding to a 612-bp fragment of the ETSTY7 ampliconic array, which is present in ECAXq17 and massively amplified in the proximal 2/3 of ECAY [Janecka et al., 2018].

\section{Fluorescence in situ Hybridization}

Delineation of the der and identification of aberration breakpoints and centromere positions was done by 2-color FISH with probes listed in Table 1 following standard protocols [Raudsepp and Chowdhary, 2008]. The probes were labeled with biotin or digoxigenin by nick translation using Biotin or DIG Nick Translation Mix (Roche Diagnostics), respectively, and the manufacturer's protocol. Hybridization signals of biotin-labeled probes were detected with Alexa Fluor ${ }^{\circledR} 488$ streptavidin conjugate (Molecular Probes, Life Technologies) and digoxigenin-labeled probes with DyLight ${ }^{\circledR} 594$ anti-digoxigenin conjugate (Vector Laboratories). Chromosomes were counterstained with 4',6-diamidino-2-phenylindole (DAPI). At least 10 cells were captured and analyzed for each experiment using a Zeiss Axioplan 2 fluorescence microscope and Isis V5.2 (MetaSystems GmbH) software.

\section{DNA Isolation and STR Genotyping}

Genomic DNA was isolated from EDTA-stabilized blood (case) with a QIAamp DNA Blood Mini Kit (Qiagen). DNA was also isolated from the sire and dam of the case using standard procedures for routine parentage testing at the Veterinary Genetics Laboratory, UC Davis. The DNA was genotyped for 15 autosomal [Khanshour et al., 2013] and 8 X-linked STRs [Anaya et al., 2017] (Table 2) using an ABI PRISM 377 (Applied Biosystems, Foster 
Table 2. X-linked STRs and their genotypes in the case and its sire and dam

\begin{tabular}{|c|c|c|c|c|c|c|c|}
\hline \multirow[t]{2}{*}{ Marker } & \multirow{2}{*}{$\begin{array}{l}\text { Genomic location, } \\
\text { ECAX, Mb, EquCab3 }\end{array}$} & \multirow{2}{*}{$\begin{array}{l}\text { Cytogenetic } \\
\text { location }\end{array}$} & \multirow{2}{*}{$\begin{array}{l}\text { NCBI } \\
\text { Accession }\end{array}$} & \multicolumn{3}{|c|}{ Genotype } & \multirow[t]{2}{*}{ Cytogenetic data } \\
\hline & & & & Case & Sire & Dam & \\
\hline LEX026 & 20.3 & $\mathrm{Xp}$ & AF075628 & $\mathrm{L}$ & $\mathrm{L}$ & $\mathrm{L}$ & Present; 2 copies \\
\hline LEX027 & 24.7 & $\mathrm{Xp}$ & AF075629 & $\mathrm{P}$ & $\mathrm{P}$ & $\mathrm{P}$ & Present; 2 copies \\
\hline TKY270 & 49.7 & Xqcen & AB048312 & OQ & $\mathrm{O}$ & OQ & Present; 2 copies \\
\hline TKY38 & 89.5 & Xq23 & AB048344 & Q & Q & Q & Deleted; 1 copy \\
\hline TKY598 & 99.2 & $\mathrm{Xq}$ & AB103816 & $\mathrm{N}$ & $\mathrm{L}$ & NO & Deleted; 1 copy \\
\hline LEX022 & 108.7 & $\mathrm{Xq}$ & AF075624 & $\mathrm{N}$ & $\mathrm{L}$ & NO & Deleted; 1 copy \\
\hline LEX003 & 114.5 & Xqdist (q27-q29) & AF075607 & $\mathrm{P}$ & $\mathrm{N}$ & $\mathrm{P}$ & Deleted; 1 copy \\
\hline TKY754 & 119.7 & Xqdist (q28-q29) & AB103972.1 & M & $\mathrm{N}$ & $\mathrm{M}$ & Deleted; 1 copy \\
\hline
\end{tabular}

City, CA, USA) following previously described methods [Juras et al., 2003] or as routinely performed for parentage testing at the Veterinary Genetics Laboratory, UC Davis.

\section{Genotyping for Deletions in the SHOX Gene}

To determine if the short stature of this case was explained by either of the 2 previously identified deletions in the short stature homeobox (SHOX) gene [Rafati et al., 2016], located within the pseudoautosomal region of X/Y, DNA from the case was genotyped using the commercially available assay (Veterinary Genetics Laboratory, UC Davis, Davis, CA, USA).

\section{Results}

\section{Karyotyping and Analysis of GTG-Banded}

Chromosomes

Karyotyping of GTG-banded metaphases revealed an abnormal diploid number of $2 n=63$ with 1 normal ECAX and 1 normal ECA16 and the presence of an abnormally large der (Fig. 1a, b). Detailed GTG-banding analysis of the der from multiple cells indicated that it is comprised of the entire Xp, X centromere, the proximal part of Xq, whereas the distal half of the long arm of the der corresponded to the entire ECA16 (Fig. 1c). The region between the Xq proximal portion and ECA16 in the der suggested the presence of more complex rearrangements, such as a possible duplication, inversion, and deletion, and was further examined by FISH analysis (see below). The abnormal karyotype was consistent in all peripheral blood lymphocytes analyzed with no evidence of mosaicism, suggesting a constitutional nature of the rearrangement.

\section{Analysis of Heterochromatic Sequences}

Close inspection of the GTG-banding pattern of the der and comparison with the banding patterns of the nor- mal ECAX and ECA16 suggested that the der may be dicentric (Fig. 1c). This was confirmed by CBG-banding, which clearly showed the presence of a single centromere in the normal ECAX and 2 distinct centromeres in the der (Fig. 2). The CBG-banding also revealed an interstitial heterochromatic band in ECAXq17, which is a known cytogenetic landmark of ECAX, corresponding at the molecular level to the equine sex chromosome specific ampliconic transcript ETSTY7 [Janecka et al., 2018]. In the der, however, there were 2 interstitial CBG-bands, suggesting a duplication (Fig. 2). The centromeric and interstitial heterochromatic patterns were further studied by FISH with horse centromeric consensus sequences ECAcons70 and ETSTY7. The results of the FISH analysis were in full agreement with CBG-banding, confirming that the der was dicentric and had a duplication involving ETSTY7 at ECAXq17 (Fig. 2).

\section{Delineation of the Derivative Chromosome by FISH}

The organization of the der was delineated by a series of dual-color FISH experiments (Fig. 3) using probes described in Table 1 . As the first step, FISH with 2 ECA16 BACs containing the PPARG and PLOD2 genes confirmed that the distal half of the long arm of the der corresponded to ECA16 (Fig. 3a, b;4). Next, FISH with BACs containing the SHOX and PLCXD1 genes from the termi-

Fig. 1. Cytogenetic analysis by GTG-banding. Arranged karyotype (a) and corresponding metaphase (b) spread showing $2 n=63$ with a single ECAX and ECA16, and a derivative (der) chromosome. c Alignment of ECAX and ECA16 with the der from 2 cells, showing that the der is comprised of Xp, X centromere, part of Xq-prox, and ECA16, including the ECA16 centromere. The region denoted with a question mark indicates complex rearrangements. Dotted lines denote centromeres and likely rearrangement breakpoints.

(For figure see next page.)
Mendoza/Schalnus/Thomson/Bellone/ Juras/Raudsepp 


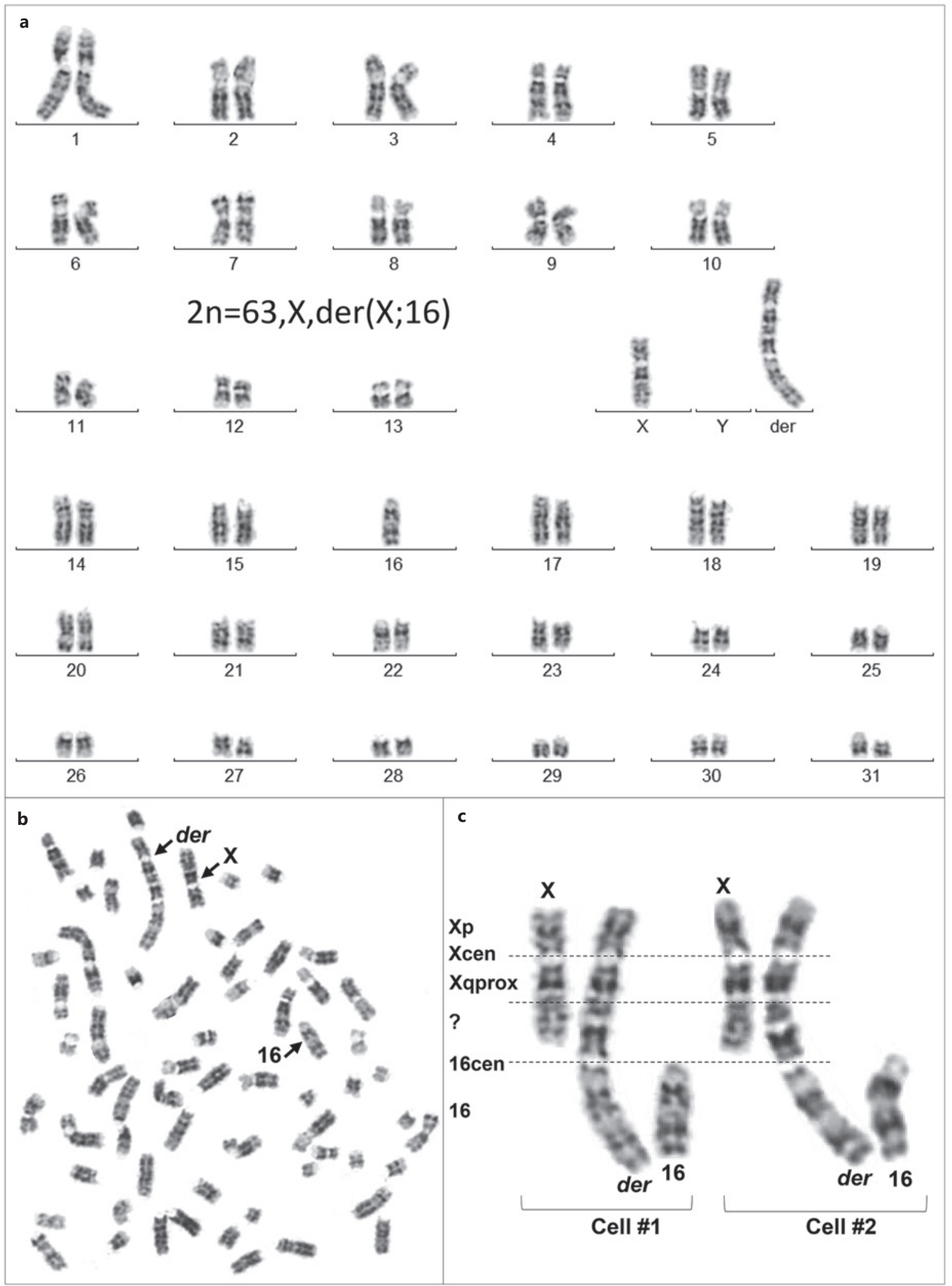


Fig. 2. Detection of centromeric and interstitial heterochromatin by CBG-banding and FISH. Two normal $\mathrm{X}$ chromosomes with CBG-banding and FISH signals (left); 2 derivative chromosomes with CBGbanding and FISH signals (middle), and ECA16 with FISH signals (right) are shown. Two-headed arrows connect corresponding regions between the chromosomes. Green signals correspond to ECAcons70. The single purple signal in normal ECAX and the upper broad purple signal in the der correspond to ETSTY7. The lower purple signals in the der and ECA16 correspond to PPARG, which was used for ECA16 identification.

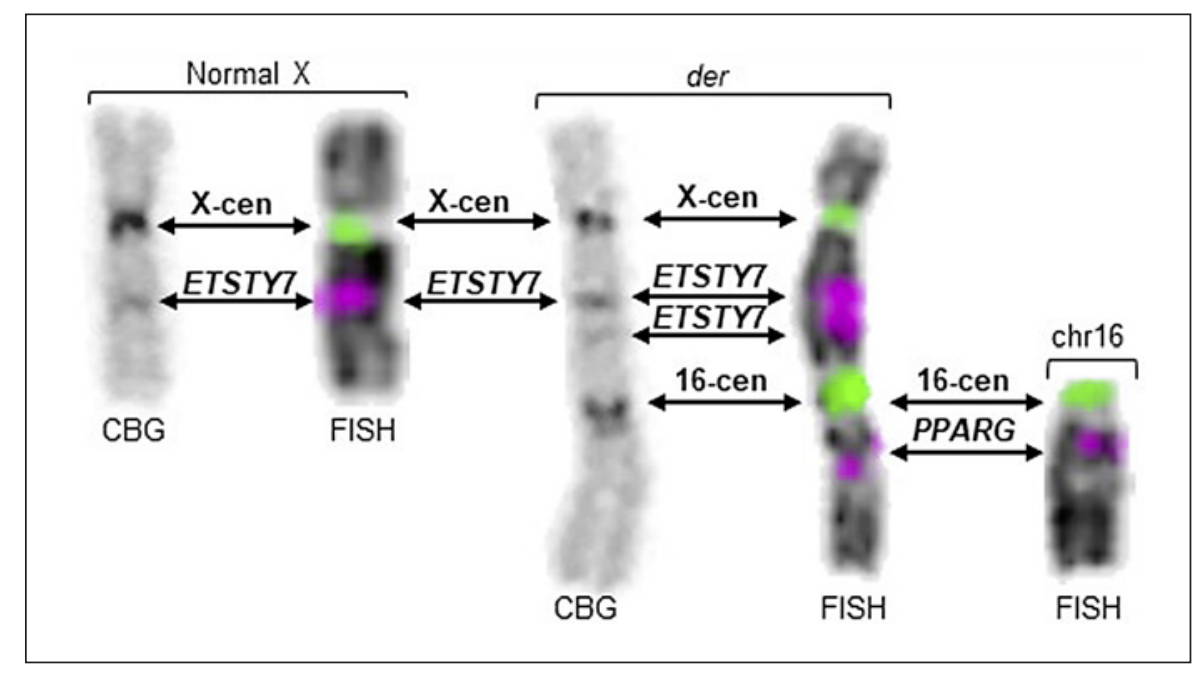

nal part of the pseudoautosomal region (PAR) at Xpter (Fig. 3c) and a BAC (121H14) from the most proximal Xp12p11 (Fig. 3d, j) showed that the short arm of the der corresponded to the entire ECAXp (Fig. 4).

However, hybridizations with BACs containing the genes SERPINA7, IRS4, PLS3, LAMP2, and TEX28 from the distal and terminal parts of Xq produced FISH signals only in the normal X but not in the der (Fig. $3 b, h, i, j$ ), indicating the deletion of Xq22q29 in the rearranged chromosome (Fig. 4). Given that the BAC containing SERPINA7 starts at $87.8 \mathrm{Mb}$ (Table 1) and the ECAXq terminal end is at $128.2 \mathrm{Mb}$ in EquCab3 (https://www. ncbi.nlm.nih.gov/genome/), we estimated the size of the $\mathrm{Xq}$ deletion in the der to be at least $40 \mathrm{Mb}$.

The proximal part of the long arm of the der, the region where GTG- and CBG-banding suggested more complex rearrangements (Fig. 1;2), was dissected with 6 ECAXq proximal markers (Table 1). All, except PCDH19, produced 2 distinct FISH signals each in metaphase (Fig. 3a, d, e, f, g). Notably, one set of signals of these markers followed their known order as cen-AMER1-ARXIST-CHM-ETSTY7-PCDH19, whereas the second set of FISH signals mirrored this orientation (Fig. 4). We concluded that the proximal part of the long arm of the der comprised a duplication and inversion, i. e., palindromic duplication of ECAXq12q21. The rearrangement breakpoint was located in a large G-negative band q21 (Fig. 4). The closest marker to the breakpoint was BAC 125B23, which contains PCDH19. FISH maps this gene to ECAXq21, and it is located at X:83.0-83.2 Mb in EquCab3 (Table 1). While in metaphase we observed a single PCDH19 FISH signal (Fig. 3g), there were 2 distinct closely located hybridization sites in the der in interphase (Fig. 3h). Given that interphase FISH resolution is 0.05-1 $\mathrm{Mb}$ [Palotie et al., 1996], we inferred that the rearrangement breakpoint in Xq21 was about $1 \mathrm{Mb}$ distal to $P C D H 19$. Because of the duplication, all genes in the ECAXq12q21 region, including XIST, had 3 copies in the genome of this Thoroughbred mare: 1 copy in the normal $\mathrm{X}$ and 2 copies in the der (Fig. 4).

Altogether, karyotyping, chromosome banding, and FISH analyses revealed that the mare had an abnormal unbalanced karyotype with a deletion of Xq22q29 and a complex $\mathrm{X} ; 16$ translocation, giving rise to a dicentric der. The latter comprised of normal Xp material, a palindromic duplication of Xq12q21, and ECA16, which was attached to the inverted duplicated segment by the centromere (Fig. 4). Based on this description and following the international cytogenetic nomenclature for chromosome aberrations in humans [ISCN, 1995], the karyotype of the mare was designated as follows: 63,X,der(X) $\operatorname{del}(\mathrm{q} 22) \operatorname{dup}(\mathrm{q} 21 \mathrm{q} 11) \mathrm{t}(\mathrm{X} ; 16)(\mathrm{q} 21 ; \mathrm{q} 11) \operatorname{dic}(\mathrm{X} ; 16)$. Though, due to the complexity of the rearrangement and to avoid ambiguity, the rearrangement is better described in words and by illustrations (Fig. 1; 4).

\section{STR Genotyping and Parental Origin of the Derivative Chromosome}

The Thoroughbred mare with the aberrant chromosome was genotyped for the standard genome-wide set of STRs confirming the parentage of her sire and dam. To determine the parental origin of the der and the efficiency of STR genotyping for the detection of X chromosome abnormalities, we genotyped the Thoroughbred mare 


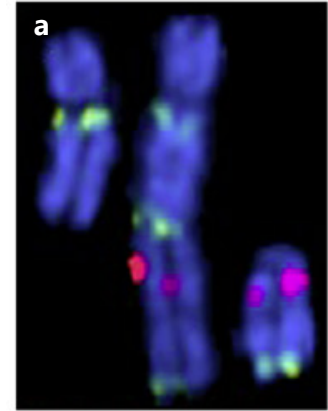

$\mathrm{Xq:155G10} \mathrm{XIST}$

16qter: 206L9 PLOD2

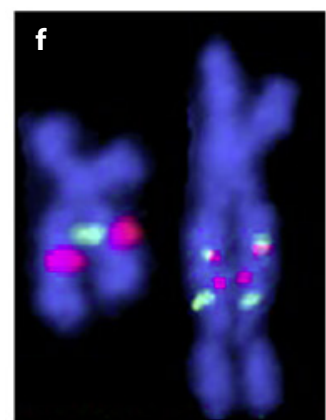

Xq:194K23 CHM Xq:ETSTY7

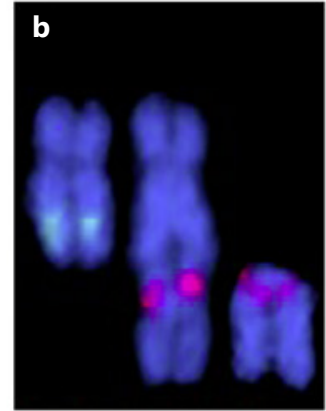

$\mathrm{Xq}: 89 \mathrm{G} 8$ LAMP2

16q:148G5 PPARG

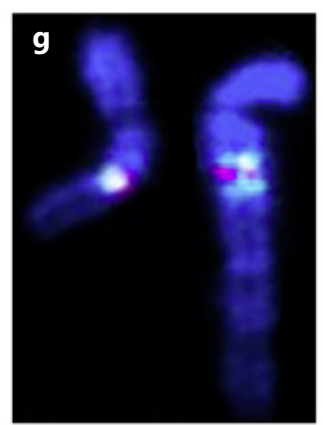

$\mathrm{Xq}:$ ETSTY7

Xq:125B23 PCDH19

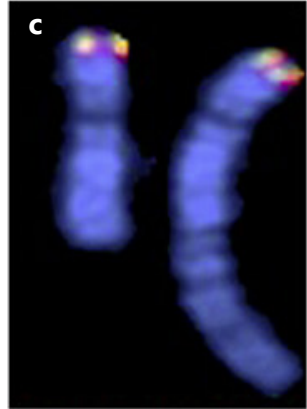

Xp:194E12 PLCXD1 Xp:288L23 SHOX

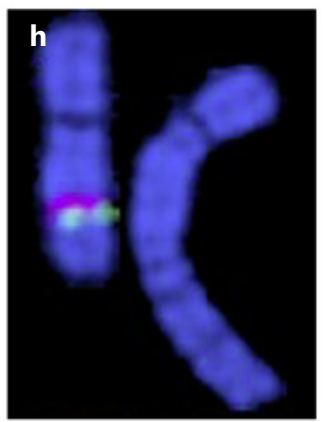

$\mathrm{Xq}: 332 \mathrm{H} 13$ PLS3

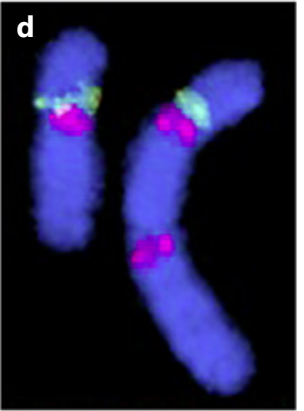

Xp:121H14 KLF8 $\mathrm{Xq}: 279 A 6$ AMER1

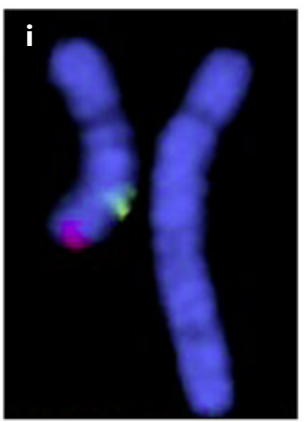

$\mathrm{Xq}: 72 J 3$ IRS4

Xq:194D2 TEX28

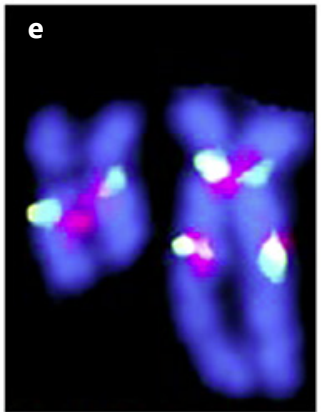

Xq:155G10 XIST

$\mathrm{Xq}: 6 \mathrm{D} 24 \mathrm{AR}$

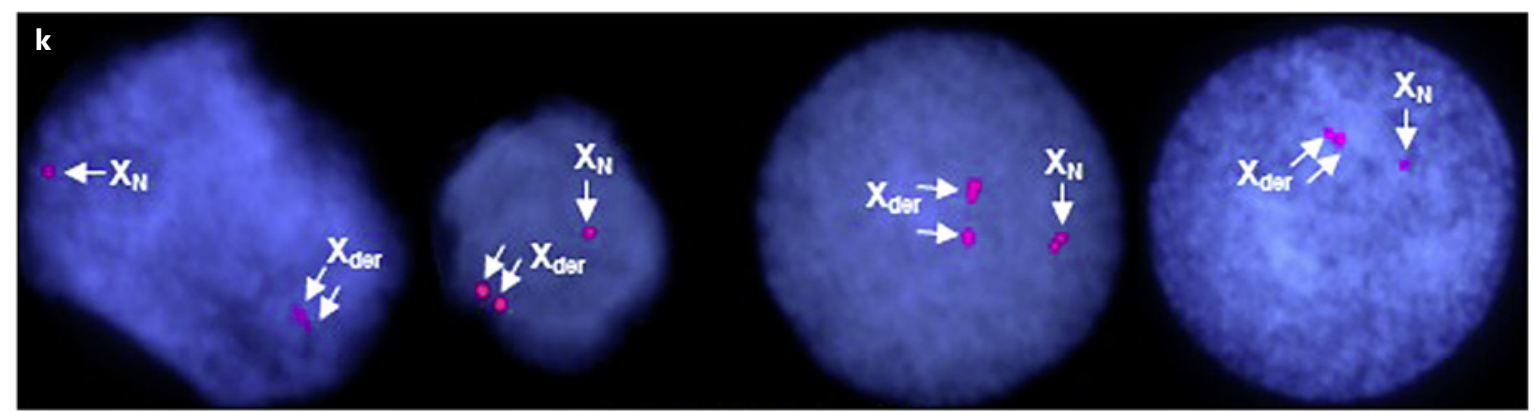

$\mathrm{Xq}: 125 \mathrm{~B} 23 \mathrm{PCDH} 19$

Fig. 3. Delineation of the derivative chromosome by FISH. a, b Dual-color FISH results with combinations of ECAX and ECA16 markers in ECAX (left), der (middle), and ECA16 (right). c-j Dual color FISH with combinations of ECAX markers in ECAX (left) and der (right). k Four interphase cells showing FISH signals with

and her sire and dam for a set of ECAX STR-markers: LEX026 and LEX027 from Xp, TKY270 from Xq proximal region, and TKY38, TKY598, LEX022, LEX003, and TKY754 spanning 89.5-119.7 Mb from the distal Xq (Table 2). All Xp markers in the trio were homozygous and

Dicentric X-Autosome Rearrangement in Horse the closest marker to the $\mathrm{Xq} 21$ breakpoint in normal $\mathrm{X}\left(\mathrm{X}_{\mathrm{N}}\right)$ and the der $\left(\mathrm{X}_{\mathrm{der}}\right)$. For each marker, chromosome arm location, BAC $\mathrm{ID}$, and a representative gene are given. Markers in green font correspond to green FISH signals and markers in red font to red FISH signals. Details for each marker are presented in Table 1.

thus uninformative. The marker from the Xq proximal region was heterozygous in the case and in agreement with cytogenetic findings that this region is present both in the normal X and the der. All markers from the distal $\mathrm{Xq}$ region were homozygous in the case, which is unex-

Cytogenet Genome Res 2020;160:597-609 
Fig. 4. Schematic summary of the complex rearrangement. G-banded ideograms [Bowling et al., 1997] of ECAX (left), ECA16 (right), and the derivative chromosome (middle). To the right of ideograms are cytogenetic locations of markers used for FISH analysis. G-positive bands are colored as follows: red, $\mathrm{Xp}$; green, $\mathrm{Xq}$ region that is duplicated and inverted in the der; black, Xq region that is deleted in the der; and blue, ECA16. Red dotted lines denote likely breakpoint regions, and arrows to the right of the der denote the palindrome of duplicated segments.

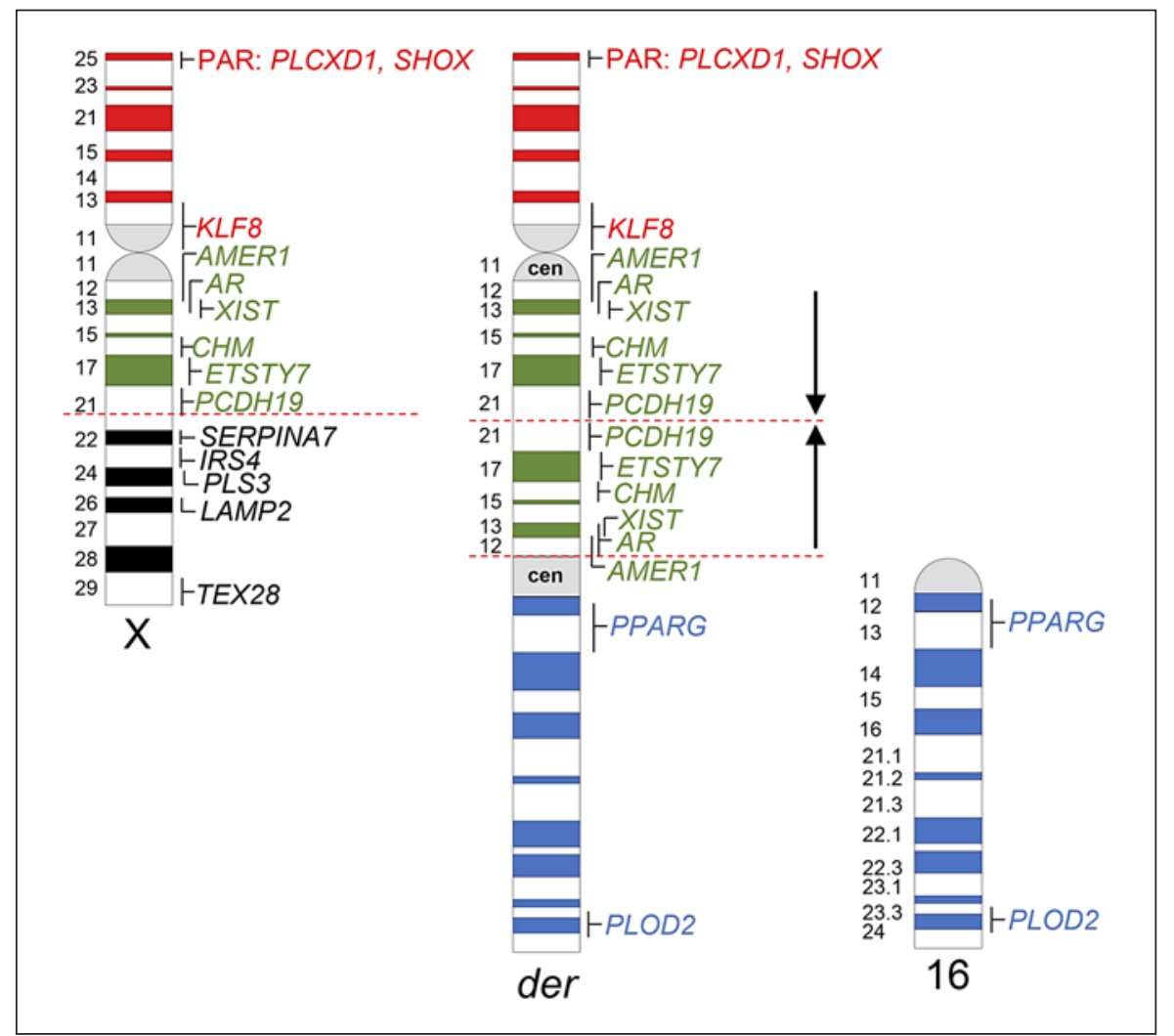

pected when compared with both mare and sire types, thus in agreement with the loss of this region in the der. Comparison of STR genotypes for the ECAXq23q29 region (deleted in the der) in the trio determined that the Thoroughbred mare carried only maternal alleles in this region (Table 2), inferring that the der was of paternal origin.

\section{SHOX Genotype}

The SHOX gene is located on the proximal portion of the $\mathrm{p}$ arm of the $\mathrm{X}$ chromosome, and previously 2 deletions in this gene were identified as causal for skeletal atavism, a recessive abnormal bone developmental disorder [Rafati et al., 2016]. While the location of this gene and copy number appear to be unaffected by the der, we aimed to rule these mutations out as the cause of the short statue in this mare. Genotyping for these deletions was performed using the commercially available assay at the Veterinary Genetics Laboratory and neither of the skeletal atavism deletions were detected (reported genotype $N / N)$.

\section{Discussion}

We described here an unusually complex unbalanced intra-X chromosome rearrangement combined with an $\mathrm{X}$-autosome translocation in an overall healthy but small in stature Thoroughbred mare. Cytogenetically, the case is unique with hardly anything comparable reported in horses or other domestic species. Of the 2 previously described $\mathrm{X}$-autosome translocations in the horse, one was balanced and reciprocal [64,X,t(1p;Xp)(1q;Xq); BugnoPoniewierska et al., 2018] and the other unbalanced [64,X,der(X),t(Xq;15q)] with Xp deletion and trisomy ECA15 [Power, 1987]. Some of the few complex X chromosome rearrangements described in humans share elements with the presented equine case, but at the same time, are also different [Haltrich et al., 2015; Villa et al., 2017; Peterson et al., 2018]. Unfortunately, as we did not have access to additional tissue samples, it was not possible to study this case for chromatin features, replication patterns, or methylation to determine the functional status of the der and its centromeres. Therefore, as follows, we will discuss the elements of this complex rearrangement one by one in the light of similar human cases and 
available information for X-autosome translocations in horses and other domestic animals.

Altogether, we analyzed almost 200 blood lymphocyte metaphase cells by GTG- and CBG-banding and FISH, observed the presence of the aberration in all, and inferred that the rearrangement was likely constitutional (nonmosaic) and of prezygotic origin. However, cytogenetic analysis of an additional tissue, if available, would be needed to confirm this conclusion.

Comparison of X chromosome STR genotypes of the case and its parents showed that the abnormal chromosome was of paternal origin (Table 2). This excludes the possibility that the sire was a carrier, because deletion of over $40 \mathrm{Mb}$ of Xq (Table 1; Fig. 4) in a male will result in nullosomy for this genomic segment and would not be compatible with life. Therefore, the rearrangements giving rise to the der must have occurred in meiosis during the sire's spermatogenesis. While STR genotyping confirmed cytogenetic findings, it also showed the limitations of this approach for discriminating between true homozygosity and hemizygosity caused by aneuploidy or deletion. For example, after initial parentage testing for the studbook registry, the case mare was identified as a possible $\mathrm{X}$ monosomy case because of homozygosity for the $\mathrm{X}$ markers tested and evidence that she was missing some $\mathrm{X}$ paternal alleles. Cytogenetic analysis refuted this, showing the presence of 2 copies of Xp material in the genome (Fig. 1; 3c, d, j). At the same time, homozygosity for all STRs in Xq22-q29 (Table 2) correctly reflected hemizygosity due to deletion of this region in the der as determined by cytogenetics (Fig. 3h, i, j). Furthermore, STR genotyping cannot detect intrachromosomal rearrangements such as inversions and duplications. Therefore, while STR genotyping is an efficient high-throughput method for initial screening of large populations for sex chromosome aneuploidies and large segmental deletions [Kakoi et al., 2005], karyotyping remains as an essential method for validation and final diagnosis.

A notable feature of the der was the presence of 2 centromeres - one being the original ECAX centromere and the other most likely the ECA16 centromere. Both centromeres were detectable by conventional CBG-banding and FISH with horse centromeric consensus sequences ECAcons70 (Fig. 2). However, these 2 methods only reveal the presence of centromeric heterochromatin composed of the tandemly repeated arrays of satellite DNA [Cerutti et al., 2016] but provide no information about centromere function. The latter is epigenetically defined by centromere-specific proteins, particularly CENPA, and can be detected by immunostaining with anti-CEN-

Dicentric X-Autosome Rearrangement in Horse
PA antibodies [Nergadze et al., 2018], which, unfortunately, was not an option for this case study. Even though dicentric chromosomes have been extensively described and studied in humans, there is no single answer for their functional regulation [Therman et al., 1986; Stimpson et al., 2012]. Both naturally occurring or engineered dicentric human chromosomes show functional plasticity, including inactivation of 1 centromere, retaining 2 functional centromeres, or having heterogeneous and clonal regulation [Higgins et al., 2005; Stimpson et al., 2012]. While the functional status of the centromeres in the dicentric der in this study remained unresolved, the mild phenotype of the carrier horse suggests that the dicentric chromosome must have successfully passed through preand postnatal mitotic cell divisions, suggesting the presence of only 1 functional centromere.

Perhaps one of the most intriguing features of this case was the distinctive short stature of $137 \mathrm{~cm}$ at the age of 2 years compared to an average height of $153 \mathrm{~cm}$ of female Thoroughbreds at this age [Green, 1976]. It is not surprising that the horse was initially flagged as a possible case of X monosomy, because short stature due to haploinsufficiency for the SHOX gene in the PAR at Xpter is one of the most characteristic features of $\mathrm{X}$ monosomy in humans [Blaschke and Rappold, 2006] and horses [Chowdhary and Raudsepp, 2000; Lear and Villagomez, 2011]. However, our cytogenetic findings and FISH analysis did not support monosomy for Xp or haploinsufficiency for SHOX (Fig. 3c; 4). In humans, some idiopathic short stature phenotypes and Leri-Weill dyschondrosteosis have been associated with microdeletions in SHOX or nearby regulatory regions [Alexandrou et al., 2016; Benito-Sanz et al., 2017]. Likewise, 2 SHOX deletions cause skeletal atavism in ponies [Rafati et al., 2016]. The condition, however, has not been found in other breeds, and unsurprisingly, the Thoroughbred mare tested negative for the skeletal atavism deletions. We therefore theorize that the observed short stature could be caused by novel SHOX mutations or mutations and/or dysregulation in other Xlinked or ECA16 genes. For example, another good Xlinked candidate for short stature is biglycan $(B G N)$. The gene encodes an extracellular matrix protein, and $B G N$ mutations cause skeletal dysplasia and dwarfism in humans [Cho et al., 2016; Meester et al., 2017]. The equine $B G N$ gene maps to chrX:126,002,378-126,018,099, the region that was deleted in the der, thus being hemizygous with any mutation exposed in the Thoroughbred mare. Alternatively, the rearrangement may have affected expression of the above mentioned or other growth-related $\mathrm{X}$-linked or ECA16 genes, which brings us to another im- 
portant point of discussion in this case - the X chromosome inactivation $(\mathrm{XCI})$.

Patterns of XCI have been extensively studied in human females carrying various $\mathrm{X}$ chromosome structural rearrangements with a general observation that $\mathrm{X}$ chromosome aberrations skew the normally random XCI [Schmidt and Du Sart, 1992; Leppig and Disteche, 2001]. In cases of intra-X chromosome rearrangements, typically the abnormal $\mathrm{X}$ is inactivated. If one $\mathrm{X}$ is involved in a balanced translocation with an autosome, the normal X is preferentially inactivated, because inactivation of the derivative chromosome may create a partially functional autosomal monosomy and $\mathrm{X}$ disomy [Schmidt and $\mathrm{Du}$ Sart, 1992; Eggermann et al., 1998; Watanabe et al., 2018]. However, unbalanced $\mathrm{X}$-autosome translocations display various XCI patterns depending on the specific features of the rearrangement [Eggermann et al., 1998]. In the majority of cases, the abnormal $\mathrm{X}$ is inactivated, and often $\mathrm{XCI}$ spreads to the autosomal region to buffer the impact of genomic imbalance of autosomal trisomy [Leppig and Disteche, 2001; Watanabe et al., 2018]. This kind of XCI pattern was also observed in the single previously reported case of unbalanced $\mathrm{X} ; 15$ translocation in the horse [Power, 1987]. However, occasionally XCI is skewed toward the normal X, and in some cases the cells retain the original random XCI [Disteche et al., 1984; Eggermann et al., 1998; Ciaccio et al., 2020].

The case described in this study involved both an unbalanced X-autosome translocation and intra-X rearrangements. This included a loss of about $40 \mathrm{Mb}$ with 530 annotated coding and RNA genes of distal Xq and a palindromic duplication of the remaining proximal Xq (Table 1; Fig. 4), containing 178 protein-coding and RNA genes (Ensembl: http://useast.ensembl.org/index.html). The latter encompassed the X inactivation center (XIC) with the XIST gene which is, as yet, not annotated in the horse genome and denoted as LOC102150010 (Table 1; https://www.ncbi.nlm.nih.gov/gene/). Even though we could not experimentally investigate XCI, the unbalanced complex dicentric X-autosome rearrangement on one hand and a very mild, almost normal phenotype on the other hand provide reasonable basis for speculations about the most plausible XCI scenarios. We are confident that inactivation of the normal $\mathrm{X}$ is unlikely, because this will cause nullosomy for the deleted $40 \mathrm{Mb}$ of Xq and cannot be compatible with cellular and organismal viability. This implies that XCI must be skewed toward the der which has 2 XICs and XIST genes and poses questions such as (1) Were both XIST copies functionally active and if so, could they interfere with each other? (2) Could 2 ac- tive XICs affect (increase/reduce) XIST RNA spreading and if so, could this interfere with XCI escape genes and their functional diploidy? For obvious reasons, these questions remained unanswered in this study and are hard to resolve even by more advanced approaches, mainly due to technical challenges to study XCI and our limited knowledge about the XCI escape genes in species other than human or mouse [Berletch et al., 2015; Tukiainen et al., 2017]. Though, a recent transcriptome analysis in a woman with a large $\mathrm{Xq}$ deletion shows that there may be mechanisms that counterbalance Xq loss by compensatory upregulation of XCI escape genes in the normal X chromosome [Santos-Reboucas et al., 2020]. Further, the overall healthy phenotype of the carrier mare allowed us to theorize that XCI most likely did not spread to the ECA16 segment of the der. This would have caused functional monosomy for an 88.9-Mb autosome with 695 coding genes (http://useast.ensembl.org/index.html) and cannot be compatible with life and normal development. It is possible that the satellite DNA of the second centromere at the X-autosome fusion site (Fig. $2 ; 4$ ) or some other sequence features of the translocation hindered the spreading of XIST RNA to ECA16. This is consistent with a similar human case with a dicentric X-autosome translocation $45, \mathrm{X}, \mathrm{dic}(\mathrm{X} ; 17)$ where the $\mathrm{X}$ portion of the abnormal chromosome was inactivated but the autosomal portion remained active [Eggermann et al., 1998]. General observations from human $\mathrm{X}$-autosome translocations are that the spreading of XCI is usually partial and patchy in the autosomal portion, suggesting that autosomal material lacks the features of the $\mathrm{X}$ chromosome that are associated with the progression and/or maintenance of XCI [Leppig and Disteche, 2001; White et al., 1998]. In conclusion, we propose that in this equine case, the normal $\mathrm{X}$ remained active and the abnormal $\mathrm{X}$ was inactive, whereas XCI did not spread into the autosomal portion of the der. Only this way the genome could have retained an almost normal genetic balance for ECA16 and Xlinked genes, which is in line with the mild phenotypic effect on the carrier.

\section{Conclusions and Future Approaches}

Complex structural abnormalities of the X chromosome, like the one described in this study, are not recurrent and occur rarely both in humans [Haltrich et al., 2015] and animals [Lear and Bailey, 2008; Lear and Villagomez, 2011; Raudsepp and Chowdhary, 2016; Szczerbal and Switonski, 2016]. At the same time, each case pro- 
vides a unique and fascinating opportunity to evaluate $\mathrm{X}$ chromosome content and inactivation status in relation to the effect on the phenotype. Like the case described here, complex X chromosome structural abnormalities result in extensive genetic imbalances of the X chromosome and autosomal material. Yet, because of XCI, compensatory upregulation of the active $\mathrm{X}$, and cell selection, these genetic imbalances can be compatible with life and, like in this equine case, have only mild phenotypes. At the same time, other forms of X chromosome structural rearrangements may cause severe developmental, reproductive, and behavioral abnormalities [Leppig and Disteche, 2001]. The effects may also vary between species, which makes every case a unique resource to acquire information about the species-specific features of $\mathrm{X}$ regulation and the role of X-linked genes in development, health, and disease. Therefore, efforts will be made to obtain additional samples for the presented case, so that cuttingedge genomics platforms could be applied to precisely determine sequence features of the rearrangement sites and the functional status of the normal and aberrant $\mathrm{X}$ chromosomes and their centromeres.

\section{Acknowledgments}

The authors are grateful to Dr. Ernie Bailey from the Gluck Equine Center, University of Kentucky, for providing access to the archive of a renowned equine cytogeneticist, the late Dr. Teri Lear (1951-2016). The documents revealed that independently from us, Dr. Lear initiated cytogenetic analysis of this case already in 2015, shortly after the horse was born. In her notes, she mentioned a pos- sible X;16 translocation but could not proceed further with the analysis. This work is dedicated to the memory of Teri Lear. The authors thank The Jockey Club for their assistance in making this study possible as well as Dr. Michael Mienaltowski, Elizabeth Esdaile, and Leslie Bickel for their technical assistance.

\section{Statement of Ethics}

Procurement of blood samples followed the United States Government Principles for the Utilization and Care of Vertebrate Animals Used in Testing, Research, and Training. These protocols were approved as AUP \#2018-0342 CA at Texas A\&M University, TX, USA.

\section{Conflict of Interest Statement}

The authors declare that they have no conflicts of interest.

\section{Funding Sources}

The study was funded by Texas A\&M Molecular Cytogenetics service and by USDA-NIFA award 2019-67015-29322.

\section{Author Contributions}

S.A.S. and B.T. provided the samples and clinical data. M.N.M. and T.R. performed cell cultures, cytogenetic and FISH analyses. R.J. and R.R.B. conducted genotyping for parental origins and mutation analysis. T.R. supervised the work and provided funding. M.N.M. and T.R. wrote the manuscript draft and all authors participated in revisions and editing the final version.

\section{References}

Abrams L, Cotter PD. Prenatal diagnosis of de novo X;autosome translocations. Clin Genet. 2004;65(5):423-8.

Alexandrou A, Papaevripidou I, Tsangaras K, Alexandrou I, Tryfonidis M, ChristophidouAnastasiadou V, et al. Identification of a novel $15.5 \mathrm{~kb}$ SHOX deletion associated with marked intrafamilial phenotypic variability and analysis of its molecular origin. J Genet. 2016;95(4):839-45.

Alkan C, Cardone MF, Catacchio CR, Antonacci F, O'Brien SJ, Ryder OA, et al. Genome-wide characterization of centromeric satellites from multiple mammalian genomes. Genome Res. 2011;21(1):137-45.

Anaya G, Molina A, Valera M, Moreno-Millán M, Azor P, Peral-García P, et al. Sex chromosomal abnormalities associated with equine infertility: Validation of a simple molecular screening tool in the purebred Spanish horse. Anim Genet. 2017;48(4):412-9.
Arrighi FE, Hsu TC. Localization of heterochromatin in human chromosomes. Cytogenetics. 1971;10(2):81-6.

Balaton BP, Brown CJ. Escape artists of the X chromosome. Trends Genet. 2016;32(6):348-59.

Basrur PK, Reyes ER, Farazmand A, King WA, Popescu PC. X-autosome translocation and low fertility in a family of crossbred cattle. Anim Reprod Sci. 2001;67(1-2):1-16.

Bellott DW, Hughes JF, Skaletsky H, Brown LG, Pyntikova T, Cho TJ, et al. Mammalian y chromosomes retain widely expressed dosage-sensitive regulators. Nature. 2014; 508(7497):494-9.

Benito-Sanz S, Belinchon-Martínez A, Aza-Carmona M, de la Torre C, Huber C, GonzálezCasado I, et al. Identification of 15 novel partial SHOX deletions and 13 partial duplications, and a review of the literature reveals intron 3 to be a hotspot region. J Hum Genet. 2017;62(2):229-34.
Berletch JB, Ma W, Yang F, Shendure J, Noble WS, Disteche CM, et al. Escape from $\mathrm{X}$ inactivation varies in mouse tissues. PLoS Genet. 2015;11(3):e1005079.

Blaschke RJ, Rappold G. The pseudoautosomal regions, SHOX and disease. Curr Opin Genet Dev. 2006;16(3):233-9.

Bowling AT, Breen M, Chowdhary BP, Hirota K, Lear T, Millon l. V, et al. ISCNH: International System for Cytogenetic Nomenclature of the Domestic Horse. Report of the third international committee for the standardization of the domestic horse karyotype, Davis, CA, USA, 1996. Committee. Chromosome Res. 1997;5:433-43.

Bugno M, Słota E, Kościelny M. Karyotype evaluation among young horse populations in Poland. Schweiz Arch Tierheilkd. 2007;149(5): $227-32$.
Dicentric X-Autosome Rearrangement in Horse
Cytogenet Genome Res 2020;160:597-609 DOI: $10.1159 / 000511236$ 
Bugno-Poniewierska M, Wojtaszek M, PawlinaTyszko K, Kowalska K, Witarski W, Raudsepp T. Evaluation of the prevalence of sex chromosome aberrations in a population of young horses - preliminary results: Doroty Russell Havemeyer 12th International Horse Genome Workshop, September 12--15, p 44.41 Pavia, Italy 2018.

Cantone I, Fisher AG. Human X chromosome inactivation and reactivation: Implications for cell reprogramming and disease. Philos Trans R Soc Lond, B, Biol Sci. 2017;372(1733).

Carrel L, Brown CJ. When the lyon (ized chromosome) roars: Ongoing expression from an inactive X chromosome. Philos Trans R Soc Lond, B, Biol Sci. 2017;372(1733).

Cerutti F, Gamba R, Mazzagatti A, Piras FM, Cappelletti E, Belloni E, et al. The major horse satellite DNA family is associated with centromere competence. Mol Cytogenet. 2016;9:35.

Cho SY, Bae JS, Kim NKD, Forzano F, Girisha $\mathrm{KM}$, Baldo C, et al. BGN mutations in Xlinked spondyloepimetaphyseal dysplasia. Am J Hum Genet. 2016;98(6):1243-8.

Chowdhary BP, Raudsepp T. Cytogenetics and physical chromosome maps, in Ruvinsky A, Bowling AT (eds): The Genetics of the Horse. Wallingford, Oxon: CAB International; 2000. p. 171-242.

Ciaccio C, Redaelli S, Bentivegna A, Marelli S, Crosti F, Sala EM, et al. Unbalanced X;autosome translocations may lead to mild phenotypes and Are associated with autoimmune diseases. Cytogenet Genome Res. 2020; 160(2):80-4.

Deng X, Berletch JB, Nguyen DK, Disteche CM. $\mathrm{X}$ chromosome regulation: Diverse patterns in development, tissues and disease. Nat Rev Genet. 2014;15(6):367-78.

Disteche CM. Escapees on the X chromosome. Proc Natl Acad Sci USA 1999;96(25):141802.

Disteche CM, Swisshelm K, Forbes S, Pagon RA. $\mathrm{X}$-inactivation patterns in lymphocytes and skin fibroblasts of three cases of X-autosome translocations with abnormal phenotypes. Hum Genet. 1984;66(1):71-6.

Eggermann T, Mau U, Klein-Vogler U, Kendziorra $\mathrm{H}$, Mackensen-Haen S, Sieverding L, et al. Molecular and cytogenetic [correction of cytogenate] analysis of an X/autosomal translocation: $45, \mathrm{X}, \operatorname{dic}(\mathrm{X} ; 17)(\mathrm{p} 22.2 ; \mathrm{p} 13) . \quad$ Clin Genet. 1998;53(4):293-7.

Green DA. Growth rate in Thoroughbred yearlings and two year olds. Equine Vet J. 1976; 8(3):133-4.

Gustavsson I, Fraccaro M, Tiepolo L, Lindsten J. Presumptive $\mathrm{X}$-autosome translocation in a cow: Preferential inactivation of the normal X chromosome. Nature. 1968;218(5137):183-4.

Haltrich I, Pikó H, Pamjav H, Somogyi A, Völgyi A, David D, et al. Complex X chromosome rearrangement associated with multiorgan autoimmunity. Mol Cytogenet. 2015;8:51
Higgins AW, Gustashaw KM, Willard HF. Engineered human dicentric chromosomes show centromere plasticity. Chromosome Res. 2005; 13(8):745-62.

ISCN: An International System for Human Cytogenetic Nomenclature (1995). S. Karger Publishers, Inc., Basel, Switzerland, 1995.

Janecka JE, Davis BW, Ghosh S, Paria N, Das PJ, Orlando L, et al. Horse Y chromosome assembly displays unique evolutionary features and putative stallion fertility genes. Nat Commun. 2018;9:2945.

Juras R, Cothran EG, Klimas R. Genetic analysis of three Lithuanian native horse breeds. Acta Agriculturae Scandinavica, Section A - Animal Science. 2003;53(4):180-5.

Kakoi H, Hirota K, Gawahara H, Kurosawa M, Kuwajima M. Genetic diagnosis of sex chromosome aberrations in horses based on parentage test by microsatellite DNA and analysis of X-and Y-linked markers. Equine Vet J. 2005;37(2):143-7.

Kalbfleisch TS, Rice ES, DePriest MS Jr., Walenz BP, Hestand MS, Vermeesch JR, et al. Improved reference genome for the domestic horse increases assembly contiguity and composition. Commun Biol. 2018;1:197.

Khanshour A, Conant E, Juras R, Cothran EG. Microsatellite analysis of genetic diversity and population structure of Arabian horse populations. J Hered. 2013;104(3):386-98.

Koykul W, Baguma-Nibasheka M, King WA, Basrur PK. Meiosis and apoptosis in germ cells of X-autosome translocation carrier boars. Mol Reprod Dev. 2000;56(4):448-57.

Lear TL, Bailey E. Equine clinical cytogenetics: The past and future. Cytogenet Genome Res. 2008;120(1-2):42-9.

Lear TL, Villagomez DAF. Cytogenetic evaluation of mares and foals, in McKinnon $\mathrm{AO}$ Vaala WE, Varner DD (eds): Equine Reproduction. Blackwell Publishing Ltd.; 2011. p. 1951-62.

Leppig KA, Disteche CM. Ring X and other structural $\mathrm{X}$ chromosome abnormalities: $\mathrm{X}$ inactivation and phenotype. Semin Reprod Med. 2001;19(2):147-57.

Loda A, Brandsma JH, Vassilev I, Servant N, Loos F, Amirnasr A, et al. Genetic and epigenetic features direct differential efficiency of Xistmediated silencing at X-chromosomal and autosomal locations. Nat Commun. 2017; 8(1):690.

Lyon MF. Gene action in the X-chromosome of the mouse (Mus musculus 1.). Nature. 1961; 190:372-3.

Lyon MF. Sex chromatin and gene action in the mammalian X-chromosome. Am J Hum Genet. 1962;14:135-48.

Meester JA, Vandeweyer G, Pintelon I, Lammens M, Van Hoorick L, De Belder S, et al. Loss-offunction mutations in the X-linked biglycan gene cause a severe syndromic form of thoracic aortic aneurysms and dissections. Genet Med. 2017;19(4):386-95.
Moyses-Oliveira M, Guilherme RS, Meloni VA, Di Battista A, de Mello CB, Bragagnolo S, et al. X-linked intellectual disability related genes disrupted by balanced $\mathrm{X}$-autosome translocations. Am J Med Genet B Neuropsychiatr Genet. 2015;168:669-77.

Nergadze SG, Piras FM, Gamba R, Corbo M, Cerutti F, McCarter JGW, et al. Birth, evolution, and transmission of satellite-free mammalian centromeric domains. Genome Res. 2018; 28(6):789-99.

Palotie A, Heiskanen M, Laan M, Horelli-Kuitunen N. High-resolution fluorescence in situ hybridization: A new approach in genome mapping. Ann Med. 1996;28(2):101-6.

Peterson JF, Basel DG, Bick DP, Chirempes B, Lorier RB, Zemlicka N, et al. A rare combination of functional disomy $\mathrm{Xp}$, deletion Xq13.2-q28 spanning the XIST gene, and duplication 3q25.33-q29 in a female with $\operatorname{der}(\mathrm{X})$ $\mathrm{t}(\mathrm{X} ; 3)(\mathrm{q} 13.2 ; \mathrm{q} 25.33)$. J Pediatr Genet. 2018; $7(1): 23-8$.

Power MM. Equine half sibs with an unbalanced $\mathrm{X} ; 15$ translocation or trisomy 28. Cytogenet Cell Genet. 1987;45(3-4):163-8.

Prueitt RL, Ross JL, Zinn AR. Physical mapping of nine $\mathrm{Xq}$ translocation breakpoints and identification of XPNPEP2 as a premature ovarian failure candidate gene. Cytogenet Cell Genet. 2000;89(1-2):44-50.

Rafati N, Andersson LS, Mikko S, Feng C, Raudsepp T, Pettersson J, et al. Large deletions at the SHOX locus in the pseudoautosomal region are associated with skeletal atavism in Shetland ponies. G3 (Bethesda). 2016;6(7): 2213-23.

Raudsepp T, Chowdhary BP. Fish for mapping single copy genes. Methods Mol Biol. 2008; 422:31-49.

Raudsepp T, Chowdhary BP. Chromosome aberrations and fertility disorders in domestic animals. Annu Rev Anim Biosci. 2016;4:15-43.

Raudsepp T, Lee EJ, Kata SR, Brinkmeyer C, Mickelson JR, Skow LC, et al. Exceptional conservation of horse-human gene order on $\mathrm{X}$ chromosome revealed by high-resolution radiation hybrid mapping. Proc Natl Acad Sci USA. 2004;101(8):2386-91.

Raudsepp T, Gustafson-Seabury A, Durkin K, Wagner ML, Goh G, Seabury CM, et al. A 4,103 marker integrated physical and comparative map of the horse genome. Cytogenet Genome Res. 2008;122(1):28-36.

Raudsepp T, Das PJ, Avila F, Chowdhary BP. The pseudoautosomal region and sex chromosome aneuploidies in domestic species. Sex Dev. 2012;6(1-3):72-83.

Santos-Reboucas CB, Boy R, Vianna EQ, Goncalves AP, Piergiorge RM, Abdala BB, et al. Skewed X-chromosome inactivation and compensatory upregulation of escape genes precludes major clinical symptoms in a female with a large Xq deletion. Front Genet. 2020;11:101 
Schmidt M, Du Sart D. Functional disomies of the $\mathrm{X}$ chromosome influence the cell selection and hence the $\mathrm{X}$ inactivation pattern in females with balanced $\mathrm{X}$-autosome translocations: A review of 122 cases. Am J Med Genet. 1992;42(2):161-9.

Seabright M. A rapid banding technique for human chromosomes. Lancet. 1971;2(7731): 971-2.

Skuse D, Printzlau F, Wolstencroft J. Sex chromosome aneuploidies. Handb Clin Neurol. 2018; 147:355-76.

Stimpson KM, Matheny JE, Sullivan BA. Dicentric chromosomes: Unique models to study centromere function and inactivation. Chromosome Res. 2012;20(5):595-605.
Szczerbal I, Switonski M. Chromosome abnormalities in domestic animals as causes of disorders of sex development or impaired fertility, in Carreira RP (ed): Insights from Animal Reproduction. INTECH, London, 2016; p. 207-25.

Therman E, Trunca C, Kuhn EM, Sarto GE. Dicentric chromosomes and the inactivation of the centromere. Hum Genet. 1986;72(3):1915.

Tukiainen T, Villani AC, Yen A, Rivas MA, Marshall JL, Satija R, et al. Landscape of X chromosome inactivation across human tissues. Nature. 2017;550(7675):244-8.
Villa N, Conconi D, Benussi DG, Tornese G, Crosti F, Sala E, et al. A complete duplication of $\mathrm{X}$ chromosome resulting in a tricentric isochromosome originated by centromere repositioning. Mol Cytogenet. 2017;10:22.

Watanabe T, Ishibashi M, Suganuma R, Ohara M, Soeda S, Komiya H, et al. Mild phenotypes associated with an unbalanced $\mathrm{X}$-autosome translocation, 46,X,der $(\mathrm{X}) \mathrm{t}(\mathrm{X} ; 8)(\mathrm{q} 28 ; \mathrm{q} 13)$. Clin Case Rep. 2018;6(8):1561-4.

White WM, Willard HF, Van Dyke DL, Wolff DJ. The spreading of $\mathrm{X}$ inactivation into autosomal material of an X;autosome translocation: Evidence for a difference between autosomal and X-chromosomal DNA. Am J Hum Genet. 1998;63(1):20-8. 